\title{
BIOWA Operators
}

\author{
Andrea Stupňanová ${ }^{(\otimes)}(\mathbb{D})$ and LeSheng $\operatorname{Jin}^{2}$ (D) \\ 1 Faculty of Civil Engineering, Slovak University of Technology in Bratislava, \\ Radlinského 11, 81005 Bratislava, Slovak Republic \\ andrea.stupnanova@stuba.sk \\ 2 Business School, Nanjing Normal University, Nanjing, China \\ j1s1980@163.com
}

\begin{abstract}
Based on bi-capacities and related Choquet integral introduced by Grabisch and Labreuche, a new generalization of OWA operators, namely BIOWA operators are introduced. Our approach is exemplified by several examples. Bi-capacities leading to the standard Yager's OWA operators on real line are completely characterized.
\end{abstract}

Keywords: Aggregation function · Bi-capacity $\cdot$ Choquet integral · OWA operator

\section{Introduction}

Bi-capacities arise as a natural generalization of capacities (fuzzy measures, normed monotone measures) in the context of decision making where underlying scales are bipolar. They, together with the related Choquet integral, are able to capture a wide variety of decision behaviours, encompassing models such as Cumulative Prospect Theory [9]. The related Choquet integral generalizes the standard extensions of the original Choquet integral, such as the asymmetric Choquet integral [3] or the symmetric (Šipoš) integral [10]. Hence we expect possible applications in all areas dealing with the aggregation of data from bipolar scales, such as expert systems [8].

In what follows, for an arbitrary $n>0$ denote $N=\{1, \ldots, n\}$. Then a set function $\mu: 2^{N} \rightarrow[0,1]$ is capacity if it is increasing and fulfils boundary conditions $\mu(\emptyset)=0$ and $\mu(N)=1$. The capacity $\mu$ is symmetric if

$$
\mu(A)=\mu(\pi(A)) \text { for any } A \in 2^{N} \text { and permutation } \pi: N \rightarrow N,
$$

$\pi(A)=\{\pi(i) \mid i \in A\}$. This symmetry holds if and only if $\mu(A)=g(\operatorname{card} A)$, where $g: N \cup\{0\} \rightarrow[0,1]$ is an increasing function fulfilling $g(0)=0$ and $g(n)=1$.

Let $f: N \rightarrow \mathbb{R}^{+}$and $\mu$ be a capacity on $N$. The Choquet integral [2] of $f$ with respect to $\mu$ is defined by

$$
\mathbf{C h}_{\mu}(f):=\int_{0}^{\infty} \mu(\{i \mid f(i) \geq t\}) \mathrm{d} t=\sum_{i=1}^{n} f(\tau(i)) \cdot\left[\mu\left(A_{\tau, i}\right)-\mu\left(A_{\tau, i+1}\right)\right],
$$


where $\tau:\{1, \ldots, n\} \rightarrow\{1, \ldots, n\}$ is any permutation such that $f(\tau(1)) \leq \cdots \leq$ $f(\tau(n)), A_{\tau, i}=\{\tau(i), \ldots, \tau(n)\}$ and $A_{\tau, n+1}:=\emptyset$. If $\mu$ is a symmetric capacity generated by $g$, then (1) can be rewritten into

$$
\mathbf{C h}_{\mu}(f)=\sum_{i=1}^{n} f(\tau(i)) \cdot[g(n-i+1)-g(n-i)] .
$$

The Choquet integral (2) is a symmetric $n$-ary aggregation function on $\mathbb{R}^{+}$, i.e., for any permutation $\pi$ it hold $\mathbf{C h}_{\mu}(f \circ \pi)=\mathbf{C h}_{\mu}(f)$, where $(f \circ \pi)(i)=$ $f(\pi(i))$. Formula $(2)$ can be applied also for inputs from $\mathbb{R}$ (corresponding to the asymmetric Choquet integral [7]).

Moreover it is enough to put $w_{i}^{g}=g(n-i+1)-g(n-i)$ to see that

$$
\mathbf{C h}_{\mu}(f)=\mathrm{OWA}_{\mathbf{w}^{g}}(f),
$$

i.e., we get an OWA operator introduced by Yager [11]. Following [7], the Choquet integral $\mathbf{C h} \mathbf{h}_{\mu}$ coincides with OWA operator if and only if $\mathbf{C h} \mathbf{h}_{\mu}$ is a symmetric aggregation function, or equivalently, if and only if $\mu$ is a symmetric capacity, $\mu(A)=g(\operatorname{card} A)$. In our contribution we apply and study the same ideas on bi-capacity-based Choquet integral [5].

The structure of this contribution is as follows. In the next section, some necessary preliminaries are given. In Sect. 3 we define a new generalization of OWA operators, BIOWA operators, based on symmetric bi-capacities. Several examples are illustrating our new approach. Finally, some concluding remarks are given.

\section{Preliminaries}

Grabisch and Labreuche [5] have introduced bi-capacities $\nu: 3^{N} \rightarrow[-1,1]$ and related Choquet integrals $[6] \mathcal{C}_{\nu}: \mathbb{R}^{n} \rightarrow \mathbb{R}$. Recall that $3^{N}=\{(A, B) \mid A, B \in$ $\left.2^{N}, A \cap B=\emptyset\right\}$. Then bi-capacity is defined as follows.

Definition 1. The function $\nu: 3^{N} \rightarrow[-1,1]$ is a bi-capacity whenever

$-\nu(N, \emptyset)=1, \nu(\emptyset, \emptyset)=0$ and $\nu(\emptyset, N)=-1$,

- $\nu$ is increasing in the first coordinate and decreasing in the second coordinate.

More, the Choquet integral $\mathcal{C}_{\nu}: \mathbb{R}^{n} \rightarrow \mathbb{R}$ is defined by

$$
\begin{aligned}
\mathcal{C}_{\nu}(f)=\sum_{i=1}^{n}|f(\sigma(i))| \cdot\left[\nu \left(A_{\sigma, i}\right.\right. & \left.\cap N_{f}^{+}, A_{\sigma, i} \cap N_{f}^{-}\right) \\
& \left.-\nu\left(A_{\sigma, i+1} \cap N_{f}^{+}, A_{\sigma, i+1} \cap N_{f}^{-}\right)\right],
\end{aligned}
$$

where $\sigma: N \rightarrow N$ is an arbitrary permutation such that

$$
|f(\sigma(1))| \leq \cdots \leq|f(\sigma(n))|
$$


$N_{f}^{+}=\{i \in N \mid f(i) \geq 0\}$ and $N_{f}^{-}=N \backslash N_{f}^{+}$.

Note that $A_{\sigma, i}=\{\sigma(i), \ldots, \sigma(n)\}$ for $i=1, \ldots, n$ and if $f \geq 0$ then $\mathcal{C}_{\nu}(f)=$ $\mathbf{C h}_{\mu}(f)$, where the right-hand side is the standard Choquet integral with respect to the capacity $\mu: 2^{N} \rightarrow[0,1], \mu(A)=\nu(A, \emptyset)$. Similarly, if $f \leq 0$ then $\mathcal{C}_{\nu}(f)=$ $-\mathbf{C h}_{\gamma}(-f)$, where $\gamma: 2^{N} \rightarrow[0,1]$ is the capacity given by $\gamma(B)=-\nu(\emptyset, B)$.

\section{BIOWA Operator}

In our contribution, we discuss symmetric Choquet integrals with respect to bicapacities, which can be seen as an important generalization of standard OWA operators on $\mathbb{R}[11]$. We will define a bi-capacity based OWA operator, BIOWA operator, as a symmetric Choquet integral $\mathcal{C}_{\nu}$, i.e. for any permutation $\pi$ and a real function $f: N \rightarrow \mathbb{R}$ it holds $\mathcal{C}_{\nu}(f)=\mathcal{C}_{\nu}(f \circ \pi)$. Clearly

$$
\left|f \circ \pi \circ \pi^{-1} \circ \sigma(1)\right| \leq \cdots \leq\left|f \circ \pi \circ \pi^{-1} \circ \sigma(n)\right|,
$$

so, for $f \circ \pi$, the role of ordering permutation is done by $\pi^{-1} \circ \sigma$, similarly $N_{f \circ \pi^{-1}}^{+}=\pi^{-1}\left(N_{f}^{+}\right), N_{f \circ \pi^{-1}}^{-}=\pi^{-1}\left(N_{f}^{-}\right)$. Based on the fact that $\mathcal{C}_{\nu}\left(\mathbf{1}_{A}-\mathbf{1}_{B}\right)=\nu(A, B)$ for any disjoint $A, B \subseteq N$, the symmetry of the Choquet integral $\mathcal{C}_{\nu}$ is equivalent to the symmetry of the related bi-capacity $\nu, \nu(A, B)=\nu\left(\pi^{-1}(A), \pi^{-1}(B)\right)$ for any permutation $\pi$. We denote $\tilde{N}=$ $\{(i, j) \mid i, j \in N \cup\{0\}, i+j \leq n\}$.

Lemma 1. A bi-capacity $\nu: 3^{N} \rightarrow[-1,1]$ is symmetric if and only if

$$
\nu(A, B)=h(\operatorname{card} A, \operatorname{card} B),
$$

where $h: \tilde{N} \rightarrow[-1,1]$

- satisfies 3 boundary conditions $h(n, 0)=1, h(0,0)=0, h(0, n)=-1$,

- is increasing in the first coordinate and

- is decreasing in the second coordinate.

Example 1. Among several interesting examples of functions $h$, we mention here the next:

(i) $h_{\mathrm{ad}}(a, b)=\frac{a-b}{n}$

(the additive $h$ )

(ii) $h_{0}(a, b)=\left\{\begin{aligned} 1 & \text { if } a=n, \\ -1 & \text { if } b=n, \\ 0 & \text { otherwise; }\end{aligned}\right.$

(iii) $h^{*}(a, b)=\left\{\begin{array}{rll}1 & \text { if } \quad a>0 \\ 0 & \text { if } \quad b<n, a=0 \\ -1 & \text { if } \quad b=n\end{array}\right.$

(the greatest $h$ ) 
(iv) $h_{*}(a, b)=\left\{\begin{array}{rll}1 & \text { if } & a=n, \\ 0 & \text { if } & a<n, b=0, \\ -1 & \text { if } & b<n ;\end{array} \quad\right.$ (the smallest $h$ )

(v) $h_{1}(a, b)=\left\{\begin{aligned} 1 & \text { if } a>0, b=0 \\ -1 & \text { if } b>0, a=0 \\ 0 & \text { otherwise }\end{aligned}\right.$

(vi) $h_{g}(a, b)=g(a)-1+g(n-b)$, where $g: N \cup\{0\} \rightarrow[0,1]$ is an increasing function fulfilling boundary conditions $g(0)=0, g(n)=1$. Note that if $g(i)=\frac{i}{n}$ then $h_{g}=h_{\text {ad }}$.

In the formula for the Choquet integral $\mathcal{C}_{\nu}(3)$, the absolute values $|f(\sigma(i))|$ of aggregated inputs are multiplied by weights

$$
w_{i}^{f}=\left[\nu\left(A_{\sigma, i} \cap N_{f}^{+}, A_{\sigma, i} \cap N_{f}^{-}\right)-\nu\left(A_{\sigma, i+1} \cap N_{f}^{+}, A_{\sigma, i+1} \cap N_{f}^{-}\right)\right] .
$$

For $f: N \rightarrow \mathbb{R}^{n}$, we define function $s_{f}: N \rightarrow\{(1,0),(0,1)\}$ by

$$
s_{f}(i):= \begin{cases}(1,0) & \text { if } \sigma(i) \in N_{f}^{+}, \\ (0,1) & \text { if } \sigma(i) \in N_{f}^{-},\end{cases}
$$

where $\sigma: n \rightarrow n$ is the unique permutation such that $|f(\sigma(1))| \leq \cdots \leq|f(\sigma(n))|$, and if for some $i<j$ it holds $|f(\sigma(i))|=|f(\sigma(j))|$ then either $\operatorname{sign} f\left(\sigma_{i}\right)=-1$ and $\operatorname{sign} f\left(\sigma_{j}\right)=1$, or $\operatorname{sign} f\left(\sigma_{i}\right)=\operatorname{sign} f\left(\sigma_{j}\right)$ and $\sigma(i)<\sigma(j)$.

Consider a symmetric bi-capacity $\nu$ described by a function $h$. Then

$$
w_{i}^{f}=h\left(\sum_{j=i}^{n} s_{f}(j)\right)-h\left(\sum_{j=i+1}^{n} s_{f}(j)\right),
$$

with convention that $\sum_{j=n+1}^{n} s_{f}(j)=(0,0)$. Then $\mathcal{C}_{\nu}(f)=\sum_{i=1}^{n} w_{i}^{f}|f(\sigma(i))|$. As we can see from (5), for a fixed $h$ the weights $w_{i}^{f}$ depend on the function $s_{f}$ only, and thus we have, in general, $2^{n}$ possible weighting vectors $\mathbf{w}_{s}=\left(w_{1}^{s}, \ldots, w_{n}^{s}\right)$,

$$
w_{i}^{s}=h\left(\sum_{j=i}^{n} s(j)\right)-h\left(\sum_{j=i+1}^{n} s(j)\right) .
$$

Now, we can define the BIOWA operator.

Definition 2. Consider a symmeric bi-capacity $\nu: 3^{N} \rightarrow[-1,1]$ described by a generating function $h$. Then BIOWA operator $\mathrm{BIOWA}_{h}: \mathbb{R}^{n} \rightarrow \mathbb{R}$ is defined as

$$
\operatorname{BIOWA}_{h}(f)=\mathcal{C}_{\nu}(f)=\sum_{i=1}^{n} w_{i}^{s}|f(\sigma(i))|
$$

where the weighting vector $\mathbf{w}_{s}$ is defined by (6) and $s=s_{f}$ is given by (4). 
Obviously, properties of the bi-polar capacities-based Choquet integrals are herited by the BIOWA operators. In particular, each BIOWA operator is an idempotent, continuous, positively homogenous and symmetric aggregation function on $\mathbb{R}[6]$.

Example 2. Let us consider $N=\{1,2,3,4,5\}$, and $f:(1,2,3,4,5) \rightarrow$ $(-4,-1,2,3,0)$. Then $\sigma=(5,2,3,4,1), N_{f}^{+}=\{3,4,5\}, N_{f}^{-}=\{1,2\}$ and $s_{f}:(1,2,3,4,5) \rightarrow((1,0),(0,1),(1,0),(1,0),(0,1))$. Then the weighting vector $\mathbf{w}_{s}$ is given by

$$
\begin{aligned}
& w_{1}^{s}=h(3,2)-h(2,2) \\
& w_{2}^{s}=h(2,2)-h(2,1) \\
& w_{3}^{s}=h(2,1)-h(1,1) \\
& w_{4}^{s}=h(1,1)-h(0,1) \\
& w_{5}^{s}=h(0,1)
\end{aligned}
$$

and the corresponding BIOWA operator is

$$
\begin{aligned}
\operatorname{BIOWA}_{h}(f) & =w_{1}^{s} \cdot 0+w_{2}^{s} \cdot 1+w_{3}^{s} \cdot 2+w_{4}^{s} \cdot 3+w_{5}^{s} \cdot 4 \\
& =h(2,2)-h(2,1)+2(h(2,1)-h(1,1))+3(h(1,1)-h(0,1))+4 h(0,1) \\
& =h(2,2)+h(2,1)+h(1,1)+h(0,1) .
\end{aligned}
$$

For $h=h_{\text {ad }}$ we get

$$
\mathrm{BIOWA}_{h_{\mathrm{ad}}}(f)=\frac{1}{5}-\frac{1}{5}=0=\frac{1}{5} \sum_{i=1}^{5} f(i) .
$$

Note that:

- if $f \geq 0$ (i.e., $N_{f}^{+}=N, N_{f}^{-}=\emptyset$ ), then the standard $\mathrm{OWA}_{\mathbf{w}^{+}}$is recovered, where $\mathbf{w}^{+}=\left(w_{1}^{+}, \ldots, w_{n}^{+}\right), w_{i}^{+}=h(n-i+1,0)-h(n-i, 0)$,

$$
\mathrm{BIOWA}_{h}(f)=\mathrm{OWA}_{\mathbf{w}^{+}}(f)=\sum_{i=1}^{n} w_{i}^{+} f(\tau(i)),
$$

where $f(\tau(1)) \leq \cdots \leq f(\tau(n))$.

- if $f<0$ (i.e., $N_{f}^{+}=\emptyset, N_{f}^{-}=N$ ), again an OWA operator is recovered, but related to the weighting vector $\mathbf{w}^{-}, w_{i}^{-}=h(0, i-1)-h(0, i), \sum_{i=1}^{5} w_{i}=1$, and then

$$
\mathrm{BIOWA}_{h}(f)=\mathrm{OWA}_{\mathbf{w}^{-}}(f)=\sum_{i=1}^{n} w_{i}^{-} f(\tau(i)) .
$$

where $f(\tau(1)) \leq \cdots \leq f(\tau(n))$. Note that this formula holds also if $f \leq 0$.

These two facts justify the name BIOWA operator. 
The BIOWA operator BIOWA $h: \mathbb{R}^{n} \rightarrow \mathbb{R}$ is a standard OWA operator of Yager if and only if $h(a, b)=h_{g}(a, b)$ from the Example 1 (vi), (i.e., $g$ generates weighting vector $\left.\mathbf{w}=\left(w_{1}, \ldots, w_{n}\right), w_{i}=g(n-i+1)-g(n-i)\right)$. Then

$$
\mathrm{BIOWA}_{h_{g}}(f)=\sum_{i=1}^{n} f(\tau(i)) \cdot(g(n-i+1)-g(n-i)),
$$

where $\tau: N \rightarrow N$ is a permutation such that $f(\tau(1)) \leq \cdots \leq f(\tau(n))$;

Example 3.

(i) Consider $h=h_{\text {ad }}$. Then

$$
\mathrm{BIOWA}_{h_{\mathrm{ad}}}(f)=\frac{1}{n} \sum_{i=1}^{n} f(i)=\operatorname{AM}(f) \quad(\text { arithmetic mean }) .
$$

(ii) Consider $h=h_{0}$. Then

$$
\mathrm{BIOWA}_{h_{0}}(f)=\operatorname{med}_{0}(f(1), \ldots, f(n))=\operatorname{med}\left(\min _{i \in N} f(i), 0, \max _{i \in N} f(i)\right) \quad(0 \text {-median }),
$$

for more details about 0 -median see [1].

(iii) Consider $h=h^{*}$. Then

$$
\operatorname{BIOWA}_{h^{*}}(f)=\max \{f(i) \mid i \in N\} .
$$

(iv) Consider $h=h_{*}$. Then

$$
\operatorname{BIOWA}_{h_{*}}(f)=\min \{f(i) \mid i \in N\} .
$$

(v) Consider $h=h_{1}$. Then

$$
\mathrm{BIOWA}_{h_{1}}(f)=\max \{f(i) \mid f(i) \geq 0\}+\min \{f(i) \mid f(i) \leq 0\} .
$$

If $n=2, f(1)=x, f(2)=y$, then

$$
\operatorname{BIOWA}_{h_{1}}(f)= \begin{cases}\max \{x, y\} & \text { if } \quad x, y \geq 0 \\ \min \{x, y\} & \text { if } x, y<0 \\ x+y & \text { otherwise }\end{cases}
$$

Remark 1. If we define dual function to $h$ by $h^{d}(a, b)=-h(b, a)$ then

$$
\mathrm{BIOWA}_{h^{d}}(f)=-\mathrm{BIOWA}_{h}(-f) \text {; }
$$

Note that $h_{\mathrm{ad}}^{d}=h_{\mathrm{ad}}$ and $h_{0}^{d}=h_{0}$, thus BIOWA $h_{\text {ad }}$ and BIOWA $h_{0}$ are homogenous. This is not the case of $h^{*}$ and $h_{*}$, as $h_{*}^{d}=h^{*} \neq h_{*}$. 


\section{Concluding Remarks}

Based on symmetric bi-capacities and related Choquet integral introduced by Grabisch and Labreuche, we have introduced and discussed BIOWA operators. These operators act just as standard OWA operator of Yager once all inputs have the same sign (but such two OWA operators may differ in weights). Symmetric bi-capacities yielding standard OWA operators being in coincidence with the related BIOWA operator are completely characterized.

We believe that the introduced BIOWA operators will find numerous applications in all areas where real data are successfully processed by means of OWA operators [4], as well as in all areas dealing with the aggregation of data from bipolar scales, such as expert systems, see [8].

Acknowledgments. The support of the grants APVV-17-0066 and VEGA 1/0006/19 is kindly announced.

\section{References}

1. Calvo, T., Kolesárová, A., Komorníková M., Mesiar, R.: Aggregation operators: properties, classes and construction methods. In: Calvo, T., Mayor, G., Mesiar, R. (eds.) Aggregation Operators: New Trends and Applications. Studies in Fuzziness and Soft Computing, vol. 97, pp. 3-104. Physica, Heidelberg (2002). https://doi. org/10.1007/978-3-7908-1787-4_1

2. Choquet, G.: Theory of capacities. Ann. Inst. Fourier 5, 131-295 (1953/1954)

3. Dennenberg, D.: Non-Additive Measure and Integral. Kluwer Academic Publishers, Dordrecht (1994)

4. Emrouznejad, A., Marra, M.: Ordered weighted averaging operators 1988-2014: a citation-based literature survey. Int. J. Intell. Syst. 29, 994-1014 (2014). https:// doi.org/10.1002/int.21673

5. Grabisch, M., Labreuche, C.: Bi-capacities-I: definition, Möbius transform and interaction. Fuzzy Sets Syst. 151, 211-236 (2005). https://doi.org/10.1016/j.fss. 2004.08.012

6. Grabisch, M., Labreuche, C.: Bi-capacities-II: the Choquet integral. Fuzzy Sets Syst. 151, 237-256 (2005)

7. Grabisch, M., Marichal, J.-L., Mesiar, R., Pap, E.: Aggregation Functions. Cambridge University Press, Cambridge (2009)

8. Heckerman, D., Shortliffe, E.: From certainty factors to belief networks. Artif. Intell. Med. 4(1), 35-52 (1992). https://doi.org/10.1016/0933-3657(92)90036-O

9. Tversky, A., Kahneman, D.: Advances in prospect theory: cumulative representation of uncertainty. J. Risk Uncertainty 5(4), 297-323 (1992). https://doi.org/10. 1007/BF00122574

10. Šipoš, J.: Integral with respect to a pre-measure. Math. Slovaca 29, 141-155 (1979)

11. Yager, R.R.: On ordered weighted averaging aggregation operators in multicriteria decisionmaking. IEEE Trans. Syst. Man Cybern. 18(1), 183-190 (1988). https:// doi.org/10.1109/21.87068 